\title{
Depression and Anxiety Correlate Differently with Salivary Free Cortisol in the Morning in Patients with Functional Somatic Syndrome
}

\author{
Hiromi Mutsuura $\cdot$ Kenji Kanbara $\cdot$ \\ Mikihiko Fukunaga · Kazumi Yamamoto · \\ Ikumi Ban · Kana Kitamura · Yoshihide Nakai
}

Published online: 7 August 2009

(c) The Author(s) 2009. This article is published with open access at Springerlink.com

\begin{abstract}
Patients presenting with functional somatic syndrome (FSS) are common, and the symptoms are persistent and difficult to treat for doctors and costly for society. The aim of this study was to clarify the common pathophysiology of FSS, especially the relationship between hypothalamic-pituitary-adrenal (HPA) axis function and psychological characteristics of patients with FSS. The subjects were 45 patients with FSS and 29 healthy controls. Salivary free cortisol was measured in the morning, and psychological tests examining depression, anxiety and quality of life (QOL) were performed on the same day. In patients with FSS, depressive scores showed a significant negative correlation with salivary free cortisol in the morning, although in healthy controls, cortisol showed a significant positive correlation with depressive scores. In addition, the correlation between other psychological test scores and cortisol secretion in patients with FSS contrasted with that of controls. The relationship between cortisol and depression, anxiety or QOL, suggests that the HPA axis of patients with FSS is dysfunctional and does not function properly when patients with FSS are under stress. This dysfunction may explain the pathology of medically unexplained persistent symptoms of patients with FSS.
\end{abstract}

Keywords Functional somatic syndrome (FSS) . Salivary free cortisol $\cdot$ Hypothalamic-pituitary-adrenal (HPA) axis · Depression · Anxiety

H. Mutsuura (更) - K. Kanbara · M. Fukunaga - K. Yamamoto ·

I. Ban · K. Kitamura · Y. Nakai

Department of Psychosomatic Medicine, Kansai Medical

University, 10-15 Fumizono-cho, Moriguchi-shi,

Osaka 570-8507, Japan

e-mail: mutsuurh@takii.kmu.ac.jp

\section{Introduction}

Functional somatic syndrome (FSS) is a group of syndromes with medically unknown origins (Wessely et al. 1999; Kirmayer and Robbins 1991; Barsky and Borus 1999). FSS is common worldwide and is observed in most areas of medicine (Henningsen et al. 2007). FSS is a serious problem because the patients are often difficult to treat, and the treatments are costly for society (Kellner 1985; Peveler et al. 1997; Barsky and Borus 1999; Wessely et al. 1999; Reid et al. 2002; Ring et al. 2004; Barsky et al. 2005; Salmon et al. 2006). FSS is characterized more by symptoms, suffering and disability, than by disease-specific, demonstrable abnormalities of structure or function (Barsky and Borus 1999). FSS is heterogeneous syndrome, but the similarities outweigh the differences (Wessely et al. 1999; Nimnuan et al. 2001). Consequently the common pathophysiology of FSS remains to be clarified.

Previously, we examined the responses of the autonomic nervous system to acute stress in patients with FSS using the psychophysiological stress profiling (PSP) test (Kanbara et al. 2004, 2005). Patients with FSS were hypo-reactive in the psychophysiological acute stress response compared with healthy controls. In addition, we reported two subgroups among patients with FSS that were identifiable based on autonomic lability (i.e. low- and high-lability) and independent of the symptoms or diagnostic category (Kanbara et al. 2007).

In general, stress increases cortisol secretion through the hypothalamic-pituitary-adrenal (HPA) axis. However, chronically stressed (Schlotz et al. 2004; Ockenfels et al. 1995; Wüst et al. 2000) and burned out (Pruessner et al. 1999) people, even though they do not require medical care, show lower salivary cortisol levels in the morning. HPA axis function in patients with FSS may show either 
hyper- reactive (Patacchioli et al. 2001; McLean et al. 2005; Geiss et al. 1997; Rief et al. 1998; Rief and Auer 2000) or hypo-reactive (Roberts et al. 2004; Jerjes et al. 2007; Böhmelt et al. 2005) patterns. These reports suggest a relationship between stress and cortisol secretion, although the relationship may not be simple higher or lower levels than controls.

Patients with FSS are under stress chronically because of medically unexplained symptoms, and are significantly more depressed than patients with known organic pathology (Henningsen et al. 2003). Patients with FSS show dysfunction in autonomic nervous system response (Kanbara et al. 2004, 2005), and if patients with FSS have HPA axis dysfunction, they may show a different cortisol secretion pattern from controls depending on the degree of the stress or exhaustion. In this study we examined the relationship between HPA axis function and the psychological characteristics of patients with FSS. Our hypothesis was that this relationship may be different in patients with FSS compared with that of healthy controls. In particular, a higher degree of depression, which may be related to exhaustion (Kopp et al. 1998; Fuhrer and Wessely 1995), would be associated with a decrease in morning free cortisol levels in patients with FSS.

\section{Methods}

\section{Subjects}

The subjects were 45 patients ( 28 females and 17 males: age range $=16-80$ years; mean \pm standard deviation $(\mathrm{SD})=$ $42.00 \pm 15.68$ years) who were diagnosed with FSS.

This study was conducted at the Department of Psychosomatic Medicine of Kansai Medical University Hospital in Osaka, Japan between August 2006 and March 2008. Patients deemed to benefit from psychophysiological assessment by the attending physicians and who had undergone PSP tests were included in the study. Patients who were unable to supply a saliva sample were excluded. A total of 67 patients were included in the study. All patients participating in the study provided written informed consent.

To define FSS, Wessely emphasized the characteristics of "medically unexplained" symptoms (Wessely et al. 1999). On the other hand, the definition provided Barsky and Borus (1999), "Functional somatic syndrome refers to several related syndromes that are characterized more by symptoms, suffering and disability than by disease-specific, demonstrable abnormalities of structure or function", may be more applicable. Based on this definition, we established the diagnostic criteria for using in our study (Kanbara et al. 2007). Patients were diagnosed as having FSS if the following three conditions were fulfilled: (1) the chief complaints were somatic symptoms that could not be explained medically or by a psychiatric disorder; (2) the subjective symptom rating score based on a visual analogue scale (VAS) was $>3 / 10$, and the duration of the symptoms was more than 6 months; and (3) the patient had disabilities in social or daily activities due to the symptoms. According to these conditions, 20 patients were excluded from the study (because of FSS clitella (1), $n=10$; VAS scale score, $n=2$; and $<6$ month duration of symptoms, $n=8$ ). One patient was excluded because of the possibility that he had Cushing syndrome, and another patient was excluded because he could not provide a saliva sample within $55 \mathrm{~min}$ after waking up [the morning salivary cortisol morning responses at $27 \pm 38 \mathrm{~min}$ after waking were assessed, as described by Yehuda et al. (2003)]. Finally, 45 patients were included for the analyses in this study.

The diagnoses of the 45 patients were functional dyspepsia $(n=6)$, irritable bowel syndrome $(n=3)$, fibromyalgia $(n=6)$, chronic fatigue syndrome $(n=2)$, chronic pain (musculoskeletal symptoms; $n=11$ ), headache (tension-type or mixed-type; $n=6$ ), other functional gastrointestinal disorder $(n=3)$, and somatoform autonomic dysfunction $(n=8)$. Diagnoses were made by the attending physicians of the patients according to each criterion of the disease. Functional dyspepsia and irritable bowel syndrome were diagnosed by the ROME III (Tack et al. 2006; Longstreth et al. 2006), fibromyalgia was diagnosed by the American College of Rheumatology 1990 Criteria (Wolfe et al. 1990), chronic fatigue syndrome was diagnosed by the criteria of the International Chronic Fatigue Syndrome Study Group (Fukuda et al. 1994), chronic pain and somatoform autonomic dysfunction was diagnosed by the International Statistical Classification of Disease and Related Health Problems Tenth Revision (World Health Organization 1992a, b), and headache was diagnosed by the International Classification of Headache Disorders 2nd Edition (Subcommittee of International Headache Society 2004). Patients with disease of HPA axis dysfunction were also excluded by the attending physicians.

Medication changes were limited as much as possible prior to the examination. There were 28 patients with FSS who took medication prior to examination. These medications included benzodiazepines $(n=18)$, antidepressants $(n=18)$, and other general medications $(n=21)$. There were no significant differences in salivary free cortisol levels in the morning between patients with FSS who did or did not take benzodiazepines $(t=-0.453, p=0.653)$, antidepressants $(t=-1.757, p=0.091)$, and general medications $(t=-0.617, p=0.541)$.

Ten patients with FSS were smokers and three patients habitually consumed alcohol. There were no significant differences in salivary free cortisol levels in the morning 
between smokers and non-smokers $(t=1.486, p=0.145)$ or habitual drinkers and non-drinkers $(t=0.328, p=$ 0.771 ) in the patient with FSS group. All subjects were advised to abstain from caffeine and alcohol intake the night before the examination and to refrain from strenuous exercise 1 day prior to the examinations.

Twenty-nine healthy subjects ( 15 female and 14 males; age range $21-64$ years; mean $\pm \mathrm{SD}=39.6 \pm 8.4$ years) served as controls. They were recruited through public announcement, and individuals receiving regular medical care or had somatic symptoms more than once a week were excluded. Subjects were paid $\$ 50$ each for their participation.

There were no significant differences between the FSS and control groups with respect to age (Mann-Whitney $U$ test; $p=0.877)$, male/female ratio $\left(\chi^{2}=1.398, \quad p=\right.$ 0.173 ), women's menstrual cycle (estrogenic phase, progestational phase, bleeding phase, or post menopause) $\left(\chi^{2}=3.386, p=0.495\right)$, and post-awaking time $(t=$ $1.723, p=0.089)$. The study was approved by the ethical committee of Kansai Medical University.

\section{Measurement of Salivary Cortisol}

Salivary cortisol was measured in the morning and PSP was examined on the same day. Measurement of salivary cortisol performed several times after waking is ideal for the evaluation of HPA axis function (Edwards et al. 2001; Kirschbaum and Hellhammer 1994, 1989; Hofman 2001; Pruessner et al. 1997). However, since our subjects were symptomatic, sampling was only performed only once at 30 min after waking, to minimize the burden on the subjects. Yehuda et al. (2003) noted that a single salivary sample taken at wake-up was a good indicator of overall cortisol production. Subjects were advised, by written and oral instructions, to refrain from eating or brushing their teeth prior to saliva sampling. Saliva was collected using Salivette sampling devices (Sarstedt, Germany). Saliva samples were gathered at the time of PSP examination, centrifuged, and then stored at $-20^{\circ} \mathrm{C}$ until assay. Salivary cortisol levels in samples were measured by SRL, Inc.

\section{Questionnaire Measures}

The beck depression inventory II (BDI-II; Beck et al. 1996) and state-trait anxiety inventory (STAI) form X (Spielberger 1966) were administered to evaluate depression and anxiety, both of which are associated with FSS (Henningsen et al. 2003). The reliability and validity of the Japanese version of the both tests were supported previously (Kojima et al. 2002; Nakazato and Mizuguchi 1982). The profile of mood state (POMS; McNair et al. 1992) was used to evaluate the temporary mood. The POMS, a 65-item scale, assesses six affective mood dimensions. The subscales consist of tension-anxiety (TA), depression-dejection (D; depressive mood or discouragement), anger-hostility (A; irritation, fury, and aggression), vigor-activity ( $\mathrm{V}$; activity, liveliness, and happiness), fatigue-inertia ( $F$; remaining inactive), and confusion-bewilderment $(\mathrm{C})$. The reliability and validity of the Japanese edition of POMS was confirmed previously (Yokoyama et al. 1990). Patients with FSS are under chronic stress, so it is important to check their actual daily life condition. The OMS 8-item short-form health survey (SF-8; Fukuhara and Suzukamo 2004) was employed to evaluate the physical and emotional quality of life (QOL).

\section{Statistical Analysis}

$T$-test, two-way factorial analysis of covariance (ANOVA) without replication and Mann-Whitney test were used to compare the salivary free cortisol in the morning and psychological test results in the FSS and control groups. The relationship between salivary free cortisol in the morning and psychological test results was analyzed by Pearson first-order correlation and multivariate regression analysis using stepwise methods. Statistical analyses were performed using SPSS 11.5 J statistical software for Windows $^{\text {TM }}$ (SPSS Inc., Chicago, IL). The two tailed $\alpha$-level was fixed at 0.05 .

\section{Results}

Table 1 shows the psychological characteristics of the FSS group and the healthy control group. In patients with FSS, the QOL scores and POMS-V were significantly lower and all the other psychological test scores were significantly higher (POMS-A was higher but not significantly) than those in healthy controls. Mean free cortisol in the morning did not differ significantly between the FSS group and control group. There were no gender differences between female and male in cortisol levels in both the FSS and control group as shown by two-way factorial ANOVA without replication (patients and controls, $F(1,70)=0.73$, $p=0.40$; female and male, $F(1,70)=0.00, p=0.97$; interaction, $F(1,70)=1.15, p=0.29)$.

Table 2 and Fig. 1 show the relationship between salivary free cortisol in the morning and psychological test scores. The relationship showed a contrasting tendency between patients with FSS and controls. In the FSS group, the BDI-II (Pearson correlation coefficient $(\mathrm{CC})=-0.37$, $p=0.013)$, POMS-TA (CC $=-0.33, p=0.025)$, POMS$\mathrm{D} \quad(\mathrm{CC}=-0.44, p=0.002)$, POMS-A $(\mathrm{CC}=-0.39$, $p=0.008), \quad$ POMS-F $(\mathrm{CC}=-0.35, \quad p=0.020) \quad$ and POMS-C $(\mathrm{CC}=-0.36, p=0.014)$ scores showed a 
Table 1 Characteristics of the FSS group and control group

\begin{tabular}{|c|c|c|c|c|c|c|c|}
\hline \multirow[b]{3}{*}{ Salivary free cortisol in the morning $(\mathrm{nM} / \mathrm{L})$} & \multicolumn{6}{|c|}{ Means $(\mathrm{SD})<$ range $>$} & \multirow{3}{*}{$\begin{array}{c}p \text { Values } \\
0.334\end{array}$} \\
\hline & \multicolumn{3}{|c|}{ FSS $(n=45)$} & \multicolumn{3}{|c|}{ Control $(n=29)$} & \\
\hline & 17.69 & $(7.16)$ & $<1.66-29.26>$ & 19.40 & $(7.46)$ & $<4.42-34.78>$ & \\
\hline Beck depression inventory II (BDI-II) & 20.53 & $(11.72)$ & $<1-47>$ & 4.83 & $(6.43)$ & $<0-23>$ & $0.000 * *$ \\
\hline \multicolumn{8}{|l|}{ State-trait anxiety inventory (STAI) } \\
\hline Trait anxiety & 55.38 & $(11.83)$ & $<25-72>$ & 39.03 & $(8.98)$ & $<28-65>$ & $0.000 * *$ \\
\hline State anxiety & 51.13 & $(11.22)$ & $<31-74>$ & 39.97 & $(6.972)$ & $<25-57>$ & $0.000 * *$ \\
\hline \multicolumn{8}{|l|}{ Profile of mood state (POMS) } \\
\hline Tension-anxiety & 55.73 & $(11.89)$ & $<33-81>$ & 42.79 & $(6.22)$ & $<33-58>$ & $0.000 * *$ \\
\hline Depression & 58.20 & $(11.58)$ & $<40-85>$ & 44.93 & $(5.28)$ & $<40-62>$ & $0.000 * *$ \\
\hline Anger-hostility & 50.71 & $(10.89)$ & $<37-81>$ & 47.76 & $(6.56)$ & $<38-61>$ & 0.124 \\
\hline Vigor & 39.29 & $(8.46)$ & $<27-59>$ & 54.86 & $(8.46)$ & $<41-68>$ & $0.000 * *$ \\
\hline Fatigue & 55.89 & $(10.66)$ & $<35-75>$ & 45.52 & $(7.23)$ & $<35-66>$ & $0.000 * *$ \\
\hline Confusion & 57.07 & $(12.63)$ & $<34-85>$ & 43.07 & $(6.04)$ & $<34-55>$ & $0.000 * *$ \\
\hline \multicolumn{8}{|l|}{ OMS 8-item short-form health survey (SF-8) } \\
\hline Physical component score (PCM) & 35.63 & $(9.15)$ & $<16.74-58.83>$ & 51.59 & $(3.85)$ & $<43.74-58.69>$ & $0.000 * *$ \\
\hline Mental component score (MCS) & 38.15 & $(8.34)$ & $<20.19-55.39>$ & 49.42 & $(5.47)$ & $<36.55-56.72>$ & $0.000 * *$ \\
\hline
\end{tabular}

** $p<0.01$

Table 2 Correlation between salivary free cortisol in the morning and psychological tests

$* * p<0.01 ; * p<0.05$

significant negative correlation with salivary free cortisol in the morning, and the SF-8 mental component score (MCS) of the QOL (CC $=-0.30, p=0.044)$ score was positively correlated. In the control group, STAI trait-anxiety $(\mathrm{CC}=0.62, p=0.000)$, STAI state-anxiety $(\mathrm{CC}=0.49$, $p=0.008)$, POMS-D (CC $=0.53, p=0.003)$, POMS-F $(\mathrm{CC}=0.39, p=0.038)$ and POMS-C $(\mathrm{CC}=0.42, p=$ $0.025)$ were correlated significantly with salivary free cortisol in the morning, and POMS-V $(\mathrm{CC}=-0.37, p=$
0.047) and MCS (CC $=-0.40, p=0.033)$ showed a significant negative correlation.

Table 3 shows the results of multivariate regression analyses using stepwise methods. In patients with FSS, POMS-D $(p \leq 0.01)$ and the FS-8 physical component score (PCS; $p=0.023$ ) showed a significant association with salivary free cortisol in the morning. In healthy controls, STAI trait anxiety $(p \leq 0.01)$ was most associated with salivary free cortisol levels in the morning. 


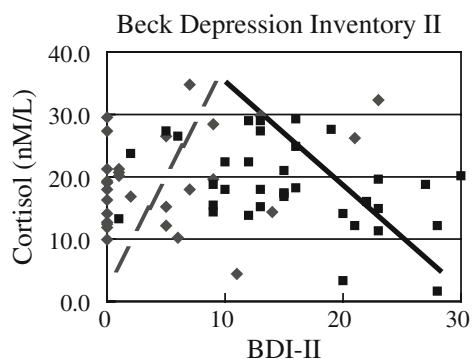

OMS Depression

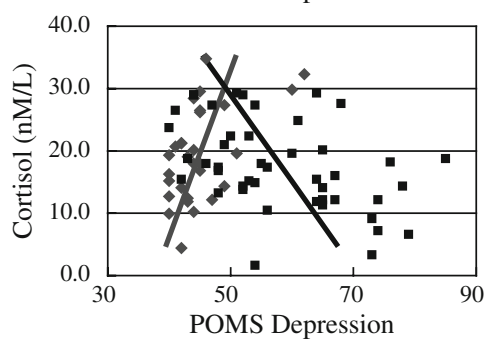

STAI trait anxiety

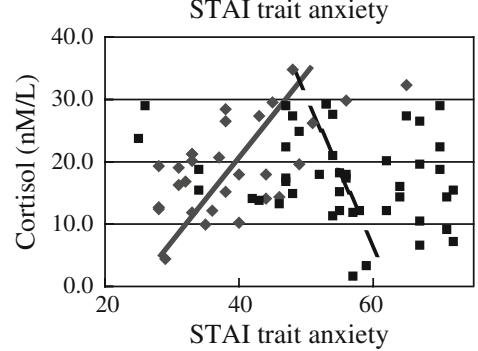

POMS Fatigue

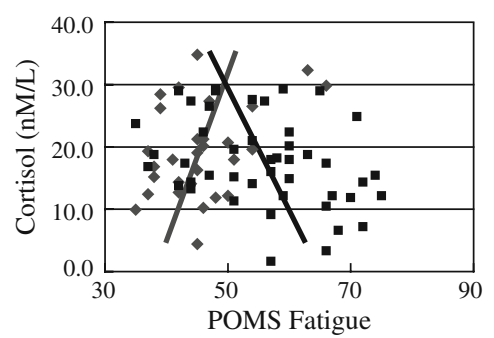

STAI state anxiety

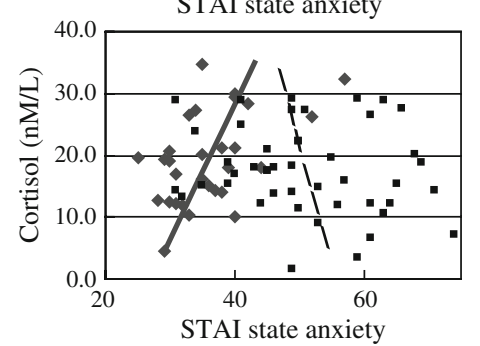

STAI state anxiety

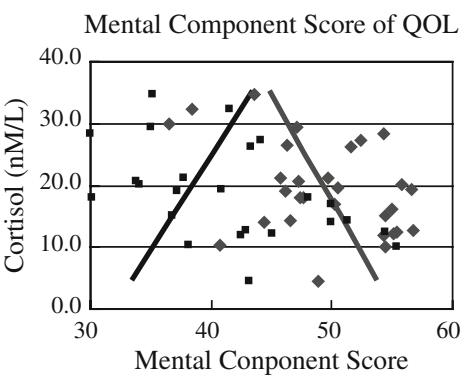

Fig. 1 The relationship between salivary free cortisol in the morning and psychological test scores. (black lines), FSS; (gray lines), Control. Solid lines are statistically significantly correlated (positively or negatively) regression lines. Dotted lines are the other regression lines. The relationship showed a contrasting tendency between patients with FSS and controls. In the relationship between cortisol and the profile of mood state (POMS) depression, fatigue and mental component score (MCS) of an 8-item short-form health survey (SF-8), the regression lines of the FSS group and the control group were crossed, and both were correlated significantly with cortisol. In the relationship between cortisol and the beck depression inventory II (BDI-II) and state-trait anxiety inventory (STAI), the FSS group and the control group show a contrasting correlation

Table 3 Multiple linear regression analysis for salivary free cortisol in the morning

\begin{tabular}{|c|c|c|c|c|c|c|}
\hline & $B$ & Standard error & $95 \%$ confidence interval & Standardizes beta & $t$ & $p$ Values \\
\hline \multicolumn{7}{|l|}{ FSS group $(n=45)$} \\
\hline (Constant) & 43.895 & 6.463 & 30.853 to 56.937 & & 6.792 & $<0.01^{* *}$ \\
\hline POMS-depression & -0.302 & 0.081 & -0.466 to -0.138 & -0.448 & -3.714 & $<0.01^{* *}$ \\
\hline SF-8 PCS & -0.242 & 0.103 & -0.450 to -0.035 & -0.31 & -2.358 & $0.023^{*}$ \\
\hline \multicolumn{7}{|c|}{ Control group $(n=29)$} \\
\hline (Constant) & -0.605 & 5.033 & -10.932 to 9.722 & & -0.120 & 0.905 \\
\hline STAI-trait anxiety & 0.512 & 0.126 & -0.254 to 0.770 & 0.617 & 4.074 & $<0.01^{* *}$ \\
\hline
\end{tabular}

POMS profile of mood state; SF 8 OMS 8-item short-form health survey; PCS physical component score; STAI state-trait anxiety inventory $* * p<0.01 ; * p<0.05$

There were no significant correlations between the duration of symptoms and salivary free cortisol in the morning in patients with FSS (min. 6 month, max. 480 month, mean 73.82 month, SD 97.41, Spearman's correlation $0.089, p$ values 0.562 ).

\section{Discussion}

The relationship between salivary free cortisol in the morning and the psychological test scores differed between patients with FSS and healthy controls (Fig. 1). In patients with FSS, the HPA axis was hyper-functional when the depressive mood state was not very high, while the HPA axis was hypo-functional when the depressive state was severe. These findings suggest that patients with FSS have a dysfunctional HPA axis, and their cortisol secretion does not function properly when patients with FSS are under stress. In this study, patients with FSS showed medically unexplained symptoms and were under prolonged stress. These characteristics may lead to exhaustion, according to the general adaptation theory of Hans Selye (1956). In our previous study, we observed psychophysiological parameters related to autonomic nervous function that were hyporeactive to mental work stress in patients with FSS (Kanbara et al. 2004, 2005), possibly because the patients 
had reached a stage of exhaustion. The HPA axis dysfunction and other dysfunctions may be characteristics of patients with FSS. The patients were unable to react properly to the stressor, which may reflect their medically unexplained symptoms.

In healthy controls, cortisol levels correlated positively with depressive mood (POMS-D). The reason that cortisol and BDI-II did not correlate in healthy controls was because 12 of the controls subjects (40\%) had a score of 0 . Therefore, the relationship between cortisol and depression scores in FSS showed a tendency contrary to that of controls.

According to the results of multivariate regression analyses in patients with FSS, the temporally depressive mood showed the strongest correlation with salivary cortisol levels in the morning. Patients with FSS suffer from unexplained physical symptoms, so it is interesting that there was a meaningful relationship between a physically low QOL (PCS) and HPA axis function. Furthermore, both depression and PCS may be related to exhaustion.

The negative correlation between the depressive state and salivary free cortisol in the morning was characteristic of patients with FSS, and not characteristic of simply depressed individuals. Parker et al. (2003) reported theoccurrence of hypercortisolism in cases of major depression. Maes et al. (1986) reported that in 100 patients with minor and major depression, the serum cortisol levels were correlated with Hamilton Depression Rate Scale scores, and cortisol levels were higher in patients with major depression than in those with minor depression. Other studies have shown that the morning free cortisol in depressed patients was higher than in controls (Yehuda et al. 1996; von Zerssen et al. 1987). In healthy young men, the morning free cortisol was reported to be increased in more depressive individuals (Pruessner et al. 2003).

One of the limitations of the present study is that some of the patients had multiple diagnoses, since FSS is a diverse syndrome. In addition, we could not ensure uniform sampling times, medication use, or menstruation cycle characteristics, any of which may have affected the data, because the subjects were experiencing symptoms that required urgent medical treatment.

Future studies are planned, with attention to the relationship between depressive mood and salivary free cortisol in the morning, that will examine the existence some subgroups in FSS.

\section{Conclusion}

The relationship between morning cortisol secretion and psychological test scores were different between patients with FSS and healthy controls. In patients with FSS, the HPA axis was hyper-functional when the depressive mood state was not very high and HPA axis was hypo-functional when the depressive state was severe. These findings suggest that patients with FSS, who were under prolonged stress with medically unexplained symptoms, have a dysfunctional HPA axis, which may be the pathology of their persistent symptoms.

Open Access This article is distributed under the terms of the Creative Commons Attribution Noncommercial License which permits any noncommercial use, distribution, and reproduction in any medium, provided the original author(s) and source are credited.

\section{References}

Barsky, A. J., \& Borus, J. (1999). Functional somatic syndromes. Annals of Internal Medicine, 130, 910-921.

Barsky, A. J., Orav, H. J., \& Bates, D. W. (2005). Somatization increases medical utilization and costs independent of psychiatric and medical comorbidity. Archives of General Psychiatry, 62(8), 903-910.

Beck, A. T., Steer, R. A., \& Brown, G. K. (1996). Manual for the beck depression inventory (2nd ed.). USA: The Psychological Corporation.

Böhmelt, A., Nater, U., Franke, S., Hellhammer, D., \& Ehlert, U. (2005). Basal and stimulated hypothalamic-pituitary-adrenal axis activity in patients with functional gastrointestinal disorders and healthy controls. Psychosomatic Medicine, 67, 288-294.

Edwards, S., Clow, A., Evans, P., \& Hucklebridge, F. (2001). Exploration of the awakening cortisol response in relation to diurnal cortisol secretory activity. Life Sciences, 68, 2093-2103.

Fuhrer, R., \& Wessely, S. (1995). The epidemiology of fatigue and depression: A French primary-care study. Psychological Medicine, 25(5), 895-905.

Fukuda, K., Staus, S. E., Hickie, I., Sharpe, M. C., Dobbins, J. G., \& Komaroff, A. (1994). The chronic fatigue syndrome: A comprehensive approach to its definition and study. International chronic fatigue syndrome study group. Annals of Internal Medicine, 121(12), 953-959.

Fukuhara, S., \& Suzukamo, Y. (2004). Manual of the SF-8 Japanese version. Kyoto: Institute for Health Outcomes \& Process Evaluation Research.

Geiss, A., Varadi, E., Steinbach, K., Bauer, H., \& Anton, F. (1997). Psychoneuroimmunological correlates of persisting sciatic pain in patients who underwent discectomy. Neuroscience Letters, 237, 65-68.

Henningsen, P., Zimmermann, T., \& Sattel, H. (2003). Medically unexplained physical symptoms, anxiety, and depression: A meta-analytic review. Psychosomatic Medicine, 65, 528-533.

Henningsen, P., Zipfel, S., \& Herzog, W. (2007). Management of functional somatic syndromes. Lancet, 369, 946-955.

Hofman, L. (2001). Human saliva as a diagnostic specimen. Journal of Nutrition, 131, 1621S-1625S.

Jerjes, W., Taylor, N., Wood, P., \& Cleare, A. (2007). Enhanced feedback sensitivity to prednisolone in chronic fatigue syndrome. Psychoneuroendocrinology, 32, 192-198.

Kanbara, K., Fukunaga, M., Mutsuura, H., Takeuchi, H., Kitamura, K., \& Nakai, Y. (2007). An exploratory study of subgrouping of patients with functional somatic syndrome based on the psychophysiological stress response: Its relationship with moods and subjective variables. Psychosomatic Medicine, 69, 158-165.

Kanbara, K., Mitani, Y., Fukunaga, M., Ishino, S., Takebayashi, N., \& Nakai, Y. (2004). Paradoxical results of psychophysiological 
stress profile in functional somatic syndrome: Correlation between subjective tension score and objective stress response. Applied Psychophysiology and Biofeedback, 29, 255-268.

Kanbara, K., Mitani, Y., Fukunaga, M., Ishino, S., Takebayashi, N., \& Nakai, Y. (2005). Characteristics of psychophysiological stress responses in patients with psychosomatic disorders. Japanese Journal of Psychosomatic Medicine, 45, 685-695.

Kellner, R. (1985). Functional somatic symptoms and hypochondriasis. A survey of empirical studies. Archives of General Psychiatry, 42, 821-833.

Kirmayer, L. J., \& Robbins, J. M. (1991). Functional somatic syndromes. In L. J. Kirmayer \& J. M. Robbins (Eds.), Current concepts of somatization: Reserch and clinical perspectives (pp. 79-106). Washington, DC: American Psychiatric Press.

Kirschbaum, C., \& Hellhammer, D. (1989). Salivary cortisol in psychobiological research: An overview. Neuropsychobiology, $22,150-169$

Kirschbaum, C., \& Hellhammer, D. (1994). Salivary cortisol in psychoneuroendocrine research: Recent developments and applications. Psychoneuroendocrinology, 19, 313-333.

Kojima, M., Furukawa, T., Takahashi, H., Kawai, M., Nagaya, T., \& Tokudome, S. (2002). Cross-cultural validation of the beck depression inventory-II in Japan. Psychiatry Research, 110, 291-299.

Kopp, M., Falger, P., Appels, A., \& Szedmak, S. (1998). Depressive symptomatology and vital exhaustion are differentially related to behavioral risk factors for coronary artery disease. Psychosomatic Medicine, 60, 752-758.

Longstreth, G. F., Thompson, W. G., Chey, W. D., Houghton, L. A., Mearin, F., \& Spiller, R. C. (2006). Functional bowel disorders. C1. Irritable bowel syndrome. Gastroenteology, 130, 1480-1481.

Maes, M., De Ruyter, M., Hobin, P., \& Suy, E. (1986). The dexamethasone suppression test, the hamilton depression rating scale and the DSM-III depression categories. Journal of Affective Disorders, 10(3), 207-214.

McLean, S., Williams, D., Harris, R., Kop, W., Groner, K., Ambrose, K., et al. (2005). Momentary relationship between cortisol secretion and symptoms in patients with fibromyalgia. Arthritis and Rheumatism, 52, 3660-3669.

McNair, D. M., Lorr, M., \& Droppleman, L. F. (1992). EITS manual for the profile of mood states (POMS). San Diego: Educational and Industrial Testing Service.

Nakazato, K., \& Mizuguchi, T. (1982). Development and validation of Japanese version of state-trait anxiety inventory. Japanese Journal of Psychosomatic Medicine, 22(2), 107-112. (Article in Japanese).

Nimnuan, C., Rabe-Hesketh, S., Wessely, S., \& Hotopf, M. (2001). How many functional somatic syndromes? Journal of Psychosomatic Research, 51, 549-557.

Ockenfels, M., Porter, L., Smyth, J., Kirschbaum, C., Hellhammer, D., \& Stone, A. (1995). Effect of chronic stress associated with unemployment on salivary cortisol: Overall cortisol levels, diurnal rhythm, and acute stress reactivity. Psychosomatic Medicine, 57, 460-467.

Parker, K., Schatzberg, A., \& Lyons, D. (2003). Neuroendocrine aspects of hypercortisolism in major depression. Hormones and Behavior, 43, 60-66.

Patacchioli, F., Angelucci, L., Dellerba, G., Monnazzi, P., \& Leri, O. (2001). Actual stress, psychopathology and salivary cortisol levels in the irritable bowel syndrome (IBS). Journal of Endocrinological Investigation, 24, 173-177.

Peveler, R., Kilkenny, L., \& Kinmonth, A. L. (1997). Medically unexplained physical symptoms in primary care: A comparison of self-report screening questionnaires and clinical opinion. Journal of Psychosomatic Research, 42, 245-252.
Pruessner, J., Hellhammer, D., \& Kirschbaum, C. (1999). Burnout, perceived stress, and cortisol responses to awakening. Psychosomatic Medicine, 61, 197-204.

Pruessner, M., Hellhammer, D., Pruessner, J., \& Lupien, S. (2003). Self-reported depressive symptoms and stress levels in healthy young men: Associations with the cortisol response to awakening. Psychosomatic Medicine, 65, 92-99.

Pruessner, J., Wolf, O., Hellhammer, D., Buske-Kirschbaum, A., von Auer, K., Jobst, S., et al. (1997). Free cortisol levels after awakening: A reliable biological marker for the assessment of adrenocortical activity. Life Sciences, 61, 2539-2549.

Reid, S., Wessely, S., Crayford, T., \& Hotopf, M. (2002). Frequent attenders with medically unexplained symptoms: Service use and costs in secondary care. British Journal of Psychiatry, 180, 248-253.

Rief, W., \& Auer, C. (2000). Cortisol and somatization. Biological Psychology, 53, 13-23.

Rief, W., Shaw, R., \& Fichter, M. (1998). Elevated levels of psychophysiological arousal and cortisol in patients with somatization syndrome. Psychosomatic Medicine, 60, 198-203.

Ring, A., Dowrick, C., Humphris, G., \& Salmon, P. (2004). Do patients with unexplained physical symptoms pressurise general practitioners for somatic treatment? A qualitative study. British Medical Journal, 328(7447), 1057.

Roberts, A., Wessely, S., Chalder, T., Papadopoulos, A., \& Cleare, A. (2004). Salivary cortisol response to awakening in chronic fatigue syndrome. British Journal of Psychiatry, 184, 136-141.

Salmon, P., Humphris, G., Ring, A., Davies, J., \& Dowrick, C. (2006). Why do primary care physicians propose medical care to patients with medically unexplained symptoms? A new method of sequence analysis to test theories of patient pressure. Psychosomatic Medicine, 68, 570-577.

Schlotz, W., Hellhammer, J., Schulz, P., \& Stone, A. (2004). Perceived work overload and chronic worrying predict weekend-weekday differences in the cortisol awakening response. Psychosomatic Medicine, 66, 207-214.

Selye, H. (1956). The stress of life. New York: McGraw-Hill.

Spielberger, C. D. (1966). Theory and research on anxiety. Anxiety and behavior. New York: Academic Press.

Subcommittee of International Headache Society. (2004). The international classification of headache disorders (2nd ed.). Cephalalgia, 24(1), 9-160.

Tack, J., Talley, N. J., Camilleri, M., Holtmann, G., Pinjin, H. U., Malagelada, J. M., et al. (2006). Functional gastroduodenal disorders. B1. Functional dyspepsia. Ganstoenteolory, 130, $1466-1468$.

von Zerssen, D., Doerr, P., Emrich, H., Lund, R., \& Pirke, K. (1987). Diurnal variation of mood and the cortisol rhythm in depression and normal states of mind. European Archives of Psychiatry and Clinical Neurolscience, 237, 36-45.

Wessely, S., Nimnuan, C., \& Sharpe, M. (1999). Functional somatic syndromes: One or many? Lancet, 354, 936-939.

Wolfe, F., Smythe, H. A., Yunas, M. B., Bennett, R. M., Bombardier, C., Golednberg, D. L., et al. (1990). The American college of rheumatology 1990 criteria for the classification of fibromyalgia. Report of the multicenter criteria committee. Arthritis and Rheum, 33(2), 160-172.

World Health Organization. (1992a). International statistical classification of disease and related health problems tenth revision. Classification of mental and behavioral disorder (pp. 166-168). Geneva: Somatoform Autonomic Dysfunction. F45-3.

World Health Organization. (1992b). International statistical classification of disease and related health problems tenth revision. Classification of mental and behavioral disorder (pp. 166-168). Geneva: Persistent Somatoform Pain Disorder. F45-4. 
Wüst, S., Federenko, I., Hellhammer, D., \& Kirschbaum, C. (2000). Genetic factors, perceived chronic stress, and the free cortisol response to awakening. Psychoneuroendocrinology, 25, 707-720.

Yehuda, R., Halligan, S., Yang, R., Guo, L., Makotkine, I., Singh, B., et al. (2003). Relationship between 24-hour urinary-free cortisol excretion and salivary cortisol levels sampled from awakening to bedtime in healthy subjects. Life Sciences, 73, 349-358.
Yehuda, R., Teicher, M., Trestman, R., Levengood, R., \& Siever, L. (1996). Cortisol regulation in posttraumatic stress disorder and major depression: A chronobiological analysis. Biological Psychiatry, 40, 79-88.

Yokoyama, K., Araki, S., Kawakami, N., \& Takeshita, T. (1990). Production of the Japanese edition of profile of mood states (POMS): Assessment of reliability and validity. Nippon Koshu Eisei Zasshi, 37(11), 913-918. (Article in Japanese). 